\title{
Second-order closure in stratified turbulence: Simulations and modeling of bulk and entrainment regions
}

\author{
L. Biferale, ${ }^{1}$ F. Mantovani, ${ }^{2}$ M. Sbragaglia, ${ }^{3}$ A. Scagliarini, ${ }^{3}$ F. Toschi,${ }^{4}$ and R. Tripiccione ${ }^{5}$ \\ ${ }^{1}$ Department of Physics and INFN, University of Tor Vergata, Via della Ricerca Scientifica 1, I-00133 Rome, Italy, and \\ Department of Physics, Eindhoven University of Technology, NL-5600 MB Eindhoven, The Netherlands \\ ${ }^{2}$ Deutsches Elektronen-Synchrotron, Platanenallee 6, D-15738 Zeuthen, Germany \\ ${ }^{3}$ Department of Physics and INFN, University of Tor Vergata, Via della Ricerca Scientifica 1, I-00133 Rome, Italy \\ ${ }^{4}$ Department of Physics and Department of Mathematics and Computer Science, Eindhoven University of Technology, \\ NL-5600 MB Eindhoven, The Netherlands, and CNR-IAC, Via dei Taurini 19, I-00185 Rome, Italy \\ ${ }^{5}$ Dipartimento di Fisica Università di Ferrara and INFN, Via G. Saragat 1, I-44100 Ferrara, Italy \\ (Received 7 January 2011; revised manuscript received 11 May 2011; published 14 July 2011)
}

\begin{abstract}
The parametrization of small-scale turbulent fluctuations in convective systems and in the presence of strong stratification is a key issue for many applied problems in oceanography, atmospheric science, and planetology. In the presence of stratification, one needs to cope with bulk turbulent fluctuations and with inversion regions, where temperature, density, or both develop highly nonlinear mean profiles due to the interactions between the turbulent boundary layer and the unmixed — stable-flow above or below it. We present a second-order closure able to cope simultaneously with both bulk and boundary layer regions, and we test it against high-resolution state-of-the-art two-dimensional numerical simulations in a convective and stratified belt for values of the Rayleigh number up to $\mathrm{Ra} \sim 10^{10}$. Data are taken from a Rayleigh-Taylor system confined by the existence of an adiabatic gradient.
\end{abstract}

DOI: 10.1103/PhysRevE.84.016305

PACS number(s): 47.20.Ma, 42.68.Bz, 47.40.-x

\section{INTRODUCTION}

Realistic parametrization of convective regions in the presence of strong stratification is a problem of interest for the evolution of the convective boundary layer in fields as different as atmospheric science [1], stellar convection [2,3], and oceanography [4-7]. The problem is intriguing also from a purely theoretical point of view: one would like to predict the interplay between buoyancy and turbulence at the edge between a convective region and a stable stratified volume above or below it. Due to turbulent activity, intermittent puffs of temperature, density, and momentum tend to enter the stably stratified region, locally producing an inversion in the energy balance: kinetic energy is indeed lost as potential energy is increased. Those turbulent puffs are the result of intense plumes traveling across the volume producing a "nonlocal" interaction between the edge and the bulk of the turbulent medium. The problem, already recognized in the early 50s [8,9], is still a subject of intense research (see, e.g., Ref. [5]). Recent studies have shown that, in the absence of strong stratification, mixing length theory based on Prandtl or Spiegel closure works very well in situations as different as the case of Rayleigh-Taylor (RT) systems or in fluid mixing by gravity currents [10-12]. Nevertheless, whenever strong stratification stops the evolution of the mixing profile, an overshooting region with temperature inversion develops and local closures fail. The problem arises from nonlocal effects caused by turbulent plumes or convective updraft.

The situation can be visualized in the middle panel of Fig. 1, where a highly turbulent configuration is confined by two stably stratified media below and above it. Similarly, in Fig. 2 we show four time snapshots focused on a typical "overshooting" event happening at the top boundary layer: here a vortex structure is injected into the stably stratified layer and sent back down to the turbulent region due to gravity restoring forces.
The system we are working with is an important variant of the classical RT case. Here, instead of isothermal mixing of two fluids with different densities we study the spatiotemporal evolution of a single-component fluid when initially prepared on the hydrostatic unstable equilibrium, i.e., with a cold uniform region in the top half and a hot uniform region in the bottom half (see right panel of Fig. 1). In the two half volumes we then fix two different homogeneous temperatures, with the corresponding hydrostatic density profiles $\partial_{z} p_{0}(z)=-g \rho_{0}(z)$. Considering that in each half we have $p_{0}(z)=T \rho_{0}(z)$, with $T$ fixed, the solution has an exponentially decaying behavior in the two half volumes, each one driven by its own temperature value. The initial hydrostatic unstable configuration is therefore given by

$$
\begin{aligned}
& T_{0}(z)=T_{u} ; \quad \rho_{0}(z)=\rho_{u} \exp \left(-g\left(z-z_{c}\right) / T_{u}\right) ; \quad z>0 ; \\
& T_{0}(z)=T_{d} ; \quad \rho_{0}(z)=\rho_{b} \exp \left(-g\left(z-z_{c}\right) / T_{d}\right) ; \quad z<0 .
\end{aligned}
$$

To be at equilibrium, we require to have the same pressure at the interface, $z=z_{c}=0$, which translates to a simple condition on the prefactor of the above expressions:

$$
\rho_{u} T_{u}=\rho_{b} T_{d} .
$$

Because $T_{u}<T_{d}$, we have at the interface $\rho_{u}>\rho_{b}$. As far as we know, there are no exhaustive detailed calculations of the stability problem for such a configuration, even though it is not too different from the usual RT compressible case [13-15]. As stated, this is not the common way to study RT systems, which is usually meant as the superposition of two different miscible fluids, isothermal, with different densities [13]. As long as compressible effects are small, one may safely neglect pressure fluctuations and write-for the case of an ideal gas- $\delta \rho / \rho \sim-\delta T / T$; the two RT experiments are then strictly equivalent. 

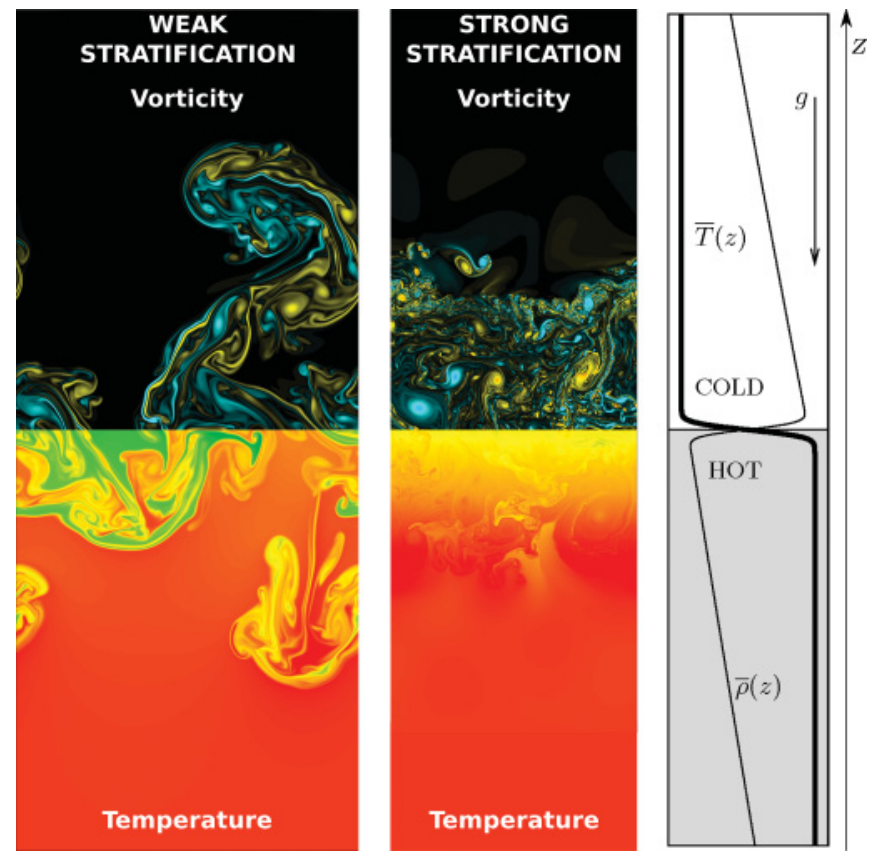

FIG. 1. (Color online) Right panel: Initial density and temperature profiles. Left and middle panels: Vorticity and temperature snapshots with weak and strong stratification, respectively. Notice the free rising plumes in the left panel typical of nonstratified RT systems. In the middle panel stratification is stronger and turbulence is confined below and above the unmixed fluid at rest: at the boundary the temperature profile overshoots.

Our configuration is obtained by evolving the above RT system in two dimensions $[16,17]$ with stratification $[18,19]$. The evolution of the RT mixing layer, unbounded in the absence of stratification [20-24], is stopped because of the presence of an adiabatic gradient: mixing is arrested when the potential temperature profile becomes flat [9]. One
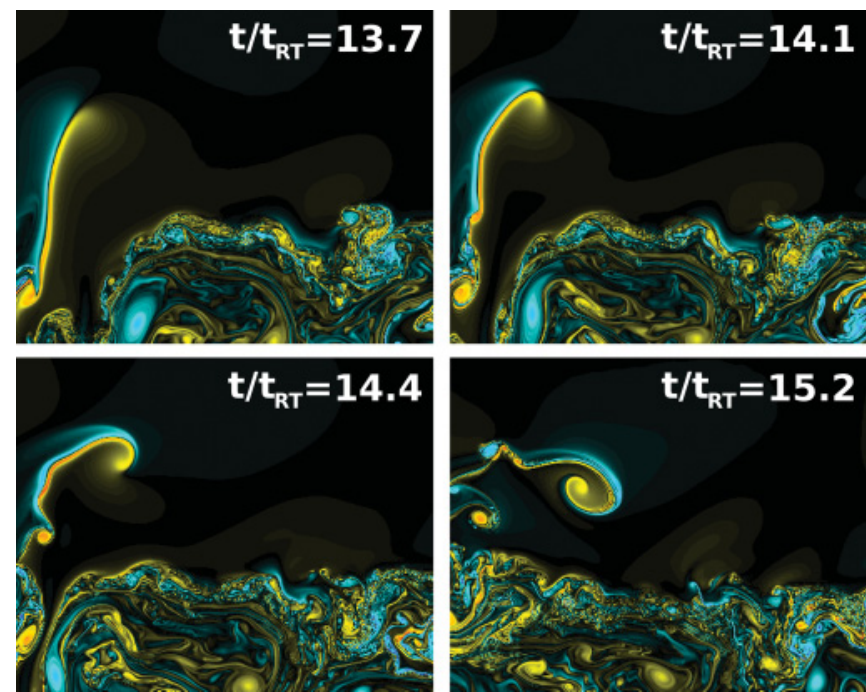

FIG. 2. (Color online) A typical overshooting event happening in the entrainment region after the mixing layer evolution stopped because of stratification (Run B). A vortex structure is injected inside the above stable region by turbulent fluctuations and then sent back by gravity. During such events, kinetic energy is lost against gravity forces.
TABLE I. Adiabatic gradient: $\gamma=-g / c_{p} ; c_{p}=2$. Adiabatic length in grid units: $L_{\gamma}=\Delta T /|\gamma|, \Delta T=T_{\text {down }}-T_{\text {up }}, \quad T_{m}=$ $\left(T_{\text {down }}+T_{\text {up }}\right) / 2$, and $T_{\text {up }}=0.95$ and $T_{\text {down }}=1.05$. Rayleigh number [9]: $\operatorname{Ra}(t)=g L(t)^{4}[\Delta T / L(t)+\gamma] /(v \kappa)$. Maximal value is obtained when $L(t)=3 / 4 L_{\gamma}$. Number of independent runs: $N_{\text {conf }}$. Characteristic time scale: $t_{\mathrm{RT}}=\sqrt{L_{x} /(g A t)}$. Atwood number $=\mathrm{At}=$ $\Delta T /\left(2 T_{m}\right)=0.05$.

\begin{tabular}{cccccccc}
\hline \hline & $L_{x}$ & $L_{z}$ & $\gamma$ & $\mathrm{Ra}_{\max }$ & $N_{\text {conf }}$ & $L_{\gamma}$ & $t_{\mathrm{RT}}$ \\
\hline A & 4096 & 10000 & $-1 \times 10^{-5}$ & $1.2 \times 10^{10}$ & 18 & 10000 & $6.4 \times 10^{4}$ \\
B & 3072 & 7200 & $-4.2 \times 10^{-5}$ & $8 \times 10^{9}$ & 11 & 2380 & $2.7 \times 10^{4}$ \\
C & 2048 & 3600 & $-8.4 \times 10^{-5}$ & $3 \times 10^{9}$ & 23 & 1190 & $1.6 \times 10^{4}$ \\
\hline \hline
\end{tabular}

control parameter is given by the Rayleigh number, i.e., the ratio between buoyancy and viscous forces in the system (see Table I). The mean temperature profile is linear in the bulk and it develops two, symmetric, overshooting regions at the edge between the turbulent boundary layer and the fluid at rest, with the heat flux inverting sign (see Fig. 3). Similar problems have recently been addressed in the realm of "standard" RT systems, for both stratified and unstratified systems.

Here we address the RT turbulent evolution for the case of an ideal gas, where the adiabatic gradient is given by $\gamma=g / c_{p}$, where $g$ is gravity and $c_{p}$ the specific heat at constant pressure.

The aim of this paper is twofold. First we present state-ofthe-art numerical results at varying stratification intensities. Second, we propose a second-order closure for the evolution of the mixing layer, able to capture both the initial transient (free of any stratification effect) and the late slowing down and stopping due to stratification effects. The main idea is to go beyond closure for the mean temperature evolution, keeping it exact and closing only for second-order quantities: the Reynolds stresses, the heat flux, and temperature fluctuations (see, e.g., Ref. [5]). We also show that our proposal goes back to the Spiegel's generalization of Prandtl mixing length theory [9] for small stratifications.

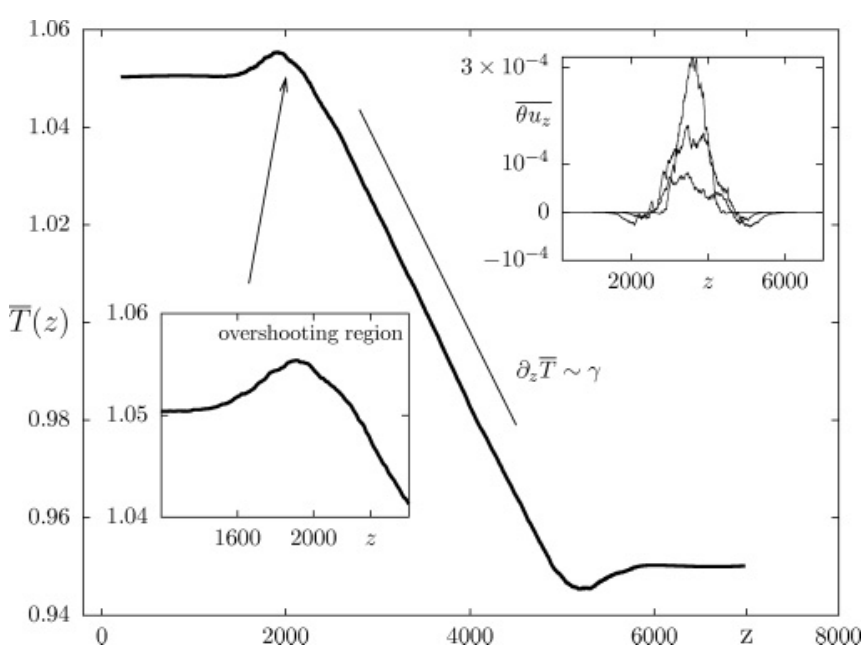

FIG. 3. Run B. Mean temperature profile $\bar{T}(z)$ after the RT evolution stopped at the adiabatic slope (solid line). Bottom left inset: Zoom of the overshooting region, with temperature inversion. Top right inset: Heat flux profile at three times, before, during, and after stopping: two regions develop with negative heat flux in correspondence with the temperature overshooting. 


\section{NUMERICAL SIMULATIONS}

We test our second-order closure against state-of-the-art numerical simulations at high resolution. We choose to work in a two-dimensional (2D) geometry to maximize the capability to get quantitative measurements at high Reynolds and Rayleigh numbers. Nevertheless, the closure here proposed should work as well in 3D geometry provided small changes are implemented (see conclusions). Choosing an RT system presents the extra complexity of nonstationarity, allowing one to probe also different turbulent time scales. Numerical simulations are performed using a recently proposed thermal lattice Boltzmann method (LBM) [25] able to reproduce the Navier-Stokes equations for momentum, density, and internal energy. Validation of the method can be found in Refs. [18,19]. Here we present numerical simulations up to $4096 \times 10000$ grid points. Table I provides details of our numerical experiment, which was run on the QPACE supercomputer [26,27]. We limit our discussion here to the case of an ideal gas, with the equation of state $P=$ $\rho T$, and at small Mach number. In this case, the main effect of stratification is limited to the presence of an adiabatic gradient affecting the evolution of temperature [9]. The equations are the following (double indexes are summed):

$$
\begin{gathered}
\partial_{t} u_{i}+u_{j} \partial_{j} u_{i}=-\frac{\partial_{i} p}{\rho_{m}}-\delta_{i, z} g \frac{\theta}{T_{m}}+v \partial_{j j} u_{i}, \quad i=x, z \\
\partial_{t} T+u_{j} \partial_{j} T-u_{z} \gamma=\kappa \partial_{j j} T
\end{gathered}
$$

where $p$ is the deviation of pressure from the hydrostatic profile, $p=P-P_{H}$ and $\partial_{z} P_{H}=-g \rho_{H}, T_{m}$ and $\rho_{m}$ are the mean temperature and density in the system, and the adiabatic gradient is given by its ideal gas expression (see Table I). In the Boussinesq approximation [9] for stratified flows, momentum is forced only by temperature fluctuations,

$$
\theta=T-\bar{T}(z)
$$

where we use the symbol $\overline{(\cdot)}$ to indicate an average over the horizontal statistically, homogeneous direction. Turbulence is triggered from the initial configuration described before by a small random perturbation of the interface between them $[18,19,28]$ (see Refs. [11,29,30] for recent studies of the classical RT system in 3D).

From Eq. (3), one easily derives the equations for the mean temperature profile,

$$
\partial_{t} \bar{T}+\partial_{z} \overline{u_{z} \theta}=\kappa \partial_{z z} \bar{T}
$$

and for the total kinetic energy, heat flux, and temperature fluctuations,

$$
\begin{aligned}
\frac{1}{2} \partial_{t} \overline{\theta^{2}}+\frac{1}{2} \partial_{z} \overline{\theta^{2} u_{z}}+\overline{u_{z} \theta}\left(\partial_{z} \bar{T}-\gamma\right) & =\kappa \overline{\theta \partial_{j j} \theta}, \\
\frac{1}{2} \partial_{t} \overline{u^{2}}+\partial_{z}\left[\overline{u^{2} u_{z}}+\overline{u_{z} p}\right] & =-g \overline{\theta u_{z}}-\epsilon_{\nu}, \\
\partial_{t} \overline{\theta u_{z}}+\partial_{z}\left[\overline{\theta u_{z}^{2}}+\overline{\theta p}\right] & =-g \overline{\theta^{2}}+\beta(z) \overline{u_{z}^{2}}-\epsilon_{\theta, u_{z}},
\end{aligned}
$$

where $u^{2}=u_{x}^{2}+u_{z}^{2}, \beta(z)=\left(\partial_{z} \bar{T}-\gamma\right)$, and the dissipative terms are $\epsilon_{\theta, u_{z}}=(v+\kappa) \overline{\partial_{i} \theta \partial_{i} u_{z}}, \epsilon_{\theta}=\kappa \overline{\partial_{i} \theta \partial_{i} \theta}$, and $\epsilon_{v}=$ $v \overline{\partial_{i} u_{j} \partial_{i} u_{j}}$. The above set of equations is exact, except for boundary dissipative contributions such as, for example, $\kappa \partial_{z} \overline{\theta \partial_{z} \theta}$, which are irrelevant when $\kappa, \nu \rightarrow 0$ in the absence of any dynamics at the walls like for RT systems. From the first of Eqs. (5) it is evident that temperature fluctuations are not forced anymore as soon as the mixing region develops a mean temperature profile with a slope of the order of the adiabatic gradient:

$$
\partial_{z} \bar{T} \sim \gamma
$$

As a consequence, from that time on, turbulence will decline. Given $\gamma$, we can identify the adiabatic length, $L_{\gamma}=\Delta T /|\gamma|$, which corresponds to the prediction for the largest possible extension of the mixing layer during the RT evolution. One possible way to define the mixing layer width, $L(t)$, is given by the integral expression $[10,22]$

$$
L(t)=2 \int d z M[\bar{c}(z)],
$$

where we have introduced the normalized temperature $c$,

$$
c(z)=\frac{T_{\text {down }}-T(z, t)}{\Delta T},
$$

and $M$ is a mixing function: $M[x]=2 x, 0 \leqslant x \leqslant 1 / 2$, and $M[x]=2(1-x), 1 / 2 \leqslant x \leqslant 1$.

In Fig. 4 we show the evolution of the mixing layer extension at changing of the intensity of the adiabatic gradient. We present data for three different stratification parameters: Run A (weak stratification), Run B, and Run C (strong stratification). Clearly, the extension of the mixing region stops growing when $L(t) \sim L_{\gamma}$.

\section{FIRST-ORDER CLOSURE}

A popular way to close the mean profile evolution is based on first-order approaches, i.e., working directly on a possible relation between heat flux and temperature gradients, so as to be able to close directly Eq. (4) and neglect the evolution of higher-order moments in Eqs. (5):

$$
\overline{u_{z} \theta}(z, t)=-K(z, t) \partial_{z} \bar{T}(z, t) .
$$

Different models have been proposed in the literature for the eddy diffusivity $K(z, t)[9,10,12]$; here we first discuss the three most popular choices:

$$
\begin{aligned}
& K^{(H)}(z, t)=k g^{1 / 2} L(t)^{3 / 2}, \quad \text { homogeneous, } \\
& K^{(P)}(z, t)=k g^{1 / 2} L(t)^{5 / 2} \partial_{z} \bar{c}, \quad \text { Prandtl, } \\
& K^{(S)}(z, t)=k g^{1 / 2} L(t)^{2}\left|\partial_{z} \bar{c}\right|^{1 / 2}, \quad \text { Spiegel, }
\end{aligned}
$$

where $k$ is a dimensionless empirical coefficient to be determined case by case. Using anyone of the above, first-order closure is very tempting because it allows for exact analytical integration of the mean profile, whenever the mixing length grows self-similarly in time. For unstratified RT situations, we know that for large time

$$
L(t) \propto g t^{2} .
$$

Inserting the above expression in Eq. (9) one gets for the normalized profile the following "diffusive" equation:

$$
\partial_{t} \bar{c}=A t^{3+2 n} \partial_{z}\left[\left(\partial_{z} \bar{c}\right)^{n} \partial_{z} \bar{c}\right]
$$



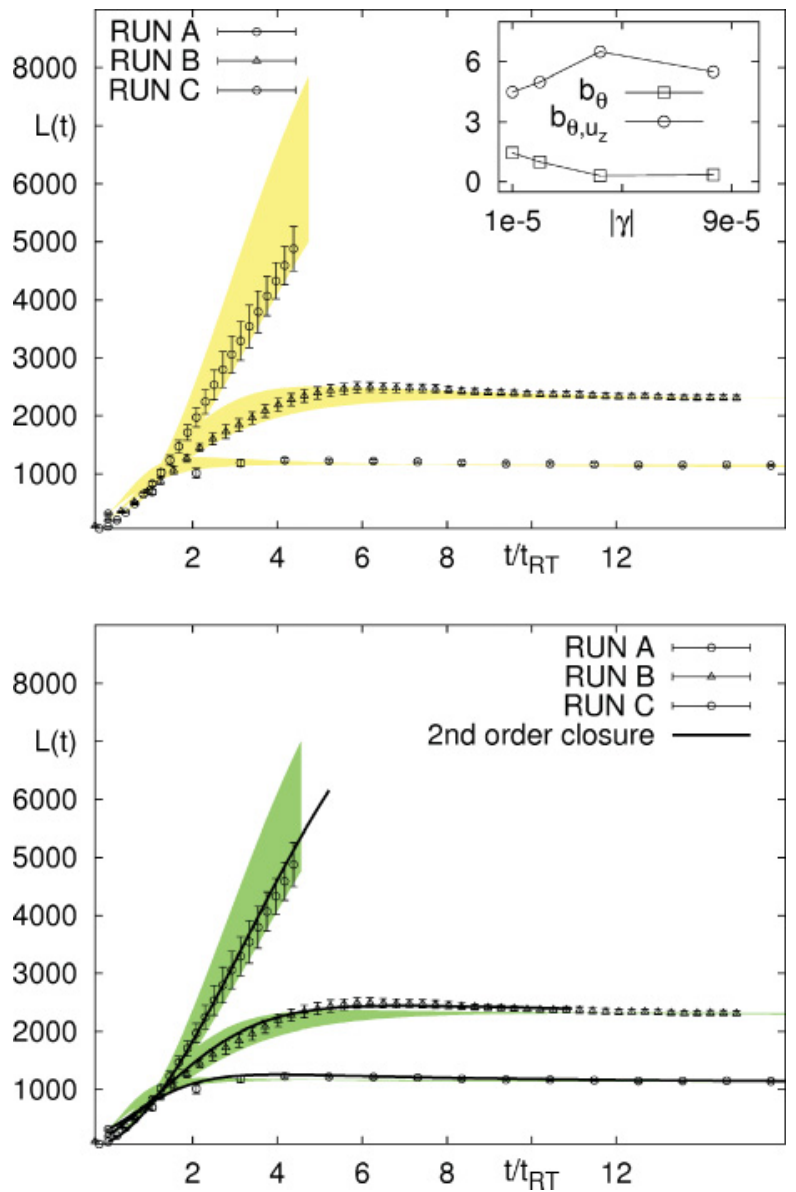

FIG. 4. (Color online) Top panel: Evolution of the mixing layer extension $L(t)$ for runs $(\mathrm{A}-\mathrm{C})$. Yellow shaded areas correspond to the second-order closure prediction for the mixing length evolution with all parameters kept fixed but $b_{\theta} \in$ [0.35:1.35], i.e., within the maximum variations needed to accurately fit also the time evolution of the whole profile at all times for all stratification values. Notice how the overall evolution of $L(t)$ is not too sensitive to the exact choice of the free parameters. Inset: Values of the best fit for the free parameters $\left(b_{\theta}, b_{\theta u_{z}}\right)$ as a function of $|\gamma|$. Bottom panel: Green shaded area corresponds to the second-order closure prediction for the mixing length evolution with all parameters kept fixed but $b_{\theta u_{z}} \in$ [4.5:6.5]. Solid lines correspond to the best fit to the mixing layer evolution taking one particular choice for the couple $\left(b_{\theta}, b_{\theta u_{z}}\right)$.

where $n$ gives the power dependency of the eddy diffusivity on the temperature gradient, i.e., $n=0$ (homogeneous), $n=1$ (Prandtl), and $n=1 / 2$ (Spiegel). The empirical coefficient $A$ is given by $A=k g^{2+n}$. Furthermore, by introducing a properly normalized temperature derivative, $\phi(z)=B \partial_{z} \bar{c}$, with $B^{n}=$ $(n+1) A /(4+2 n)$, and a rescaled time variable $t^{\prime}=t^{2(2+n)}$, one ends with a standard form $[10,31,32]$ :

$$
\partial_{t^{\prime}} \phi=\partial_{z}\left(\phi^{n} \partial_{z} \phi\right) .
$$

It is easy to realize that using the homogeneous eddy diffusivity case, $n=0$, leads to the well-known error function solution for the mean temperature profile. An exact solution can be found also for the eddy diffusivities based on the Prandtl mixing length theory or on Spiegel's approach, i.e., second and third expressions in Eq. (9). Introducing the normalized variable $\xi=z / z_{1}(t)$, the solutions for the two cases are [31]

$$
\begin{aligned}
c^{(P)}(\xi)= & \frac{3}{4}\left(\xi-\frac{1}{3} \xi^{3}+\frac{2}{3}\right) ; \\
c^{(S)}(\xi)= & \frac{15}{16}\left(\xi-\frac{2}{3} \xi^{3}+\frac{1}{5} \xi^{5}+\frac{8}{15}\right) ; \\
& -1 \leqslant \xi \leqslant 1 .
\end{aligned}
$$

Where $z_{1}(t)$ is the semiextension of the mixing layer; i.e., it has been chosen such that the profile derivative vanishes at the two extrema $\phi\left[ \pm z_{1}(t)\right]=0$. In particular, we have for the Prandtl case $z_{1}(t)=\delta g t^{2}$, with $\delta=(3 / 2 A)^{1 / 3}[10]$, while for the Spiegel's case we have $z_{1}(t)=\delta g t^{2}$, with $\delta=\left(A^{2} 81 / 160\right)^{1 / 5}$.

All these attempts work well and are almost indistinguishable in the absence of overshooting as one can see in Fig. 5, where we plot both the normalized mean temperature profile and its derivative for a given time during the RT evolution of Run A superposed with the two predictions using either Prandtl's or Spiegel's closure. In the same figure we also plot the prediction for the second-order closure we develop in the next section. The above procedure cannot be extended to the case where the mixing length does not
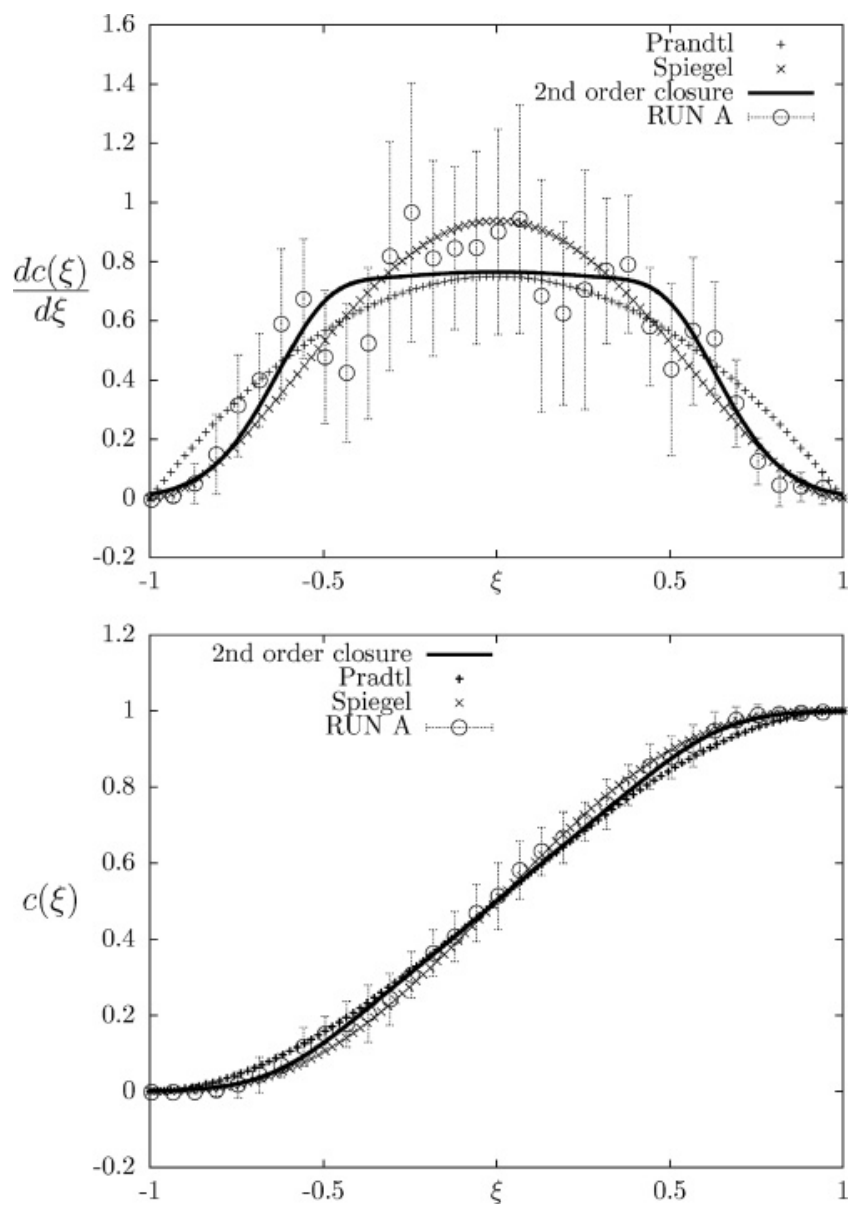

FIG. 5. Run A, small stratification. Bottom panel: (Circles) Normalized mean temperature profile, $c(\xi)$, as a function of the normalized distance from the center of the mixing layer, $\xi$, at $t=4 t_{\mathrm{RT}}$. $(+)$ The same for Prandtl closure $c^{(P)}(\xi) .(\times)$ The same for Spiegel closure $c^{(S)}(\xi)$. Solid line: Our second-order closure. Top panel: The same data sets for the mean derivative of the profile, $\frac{d c(\xi)}{d \xi}$. 
evolve self-similarly in time, i.e., when stratifications stops the evolution. This is because of two reasons. First, in this case the temporal evolution of $L(t)$ is not known analytically, being itself dependent on the whole evolution of the profile via Eq. (7), leading to an integrodifferential equation for the temperature profile. Second, the presence of an overshooting with temperature and heat flux inversion suggests that simple approaches based on eddy diffusivity may become unstable. This is why we develop a second-order closure in the next section.

\section{SECOND-ORDER CLOSURE}

The overshoot developing at the edge between turbulent and unmixed fluid is visible in Fig. 3, where we show both the nonlinear temperature inversion (inset bottom left) and the corresponding inversion in the heat flux (inset top right). This overshooting region is clearly a problem for any attempt to close the mean profile evolution with any sort of local eddy diffusivity as developed in the previous section. Inversion in temperature and heat flux implies a negative effective eddy diffusivity and the closure becomes unstable. In order to overcome this difficulty, we keep exact the equations for the mean profile (4) and close only the fluctuations at the second-order moments in Eqs. (5). Considering $u_{z} \sim u$, we are left with six unknown to be defined: the three dissipative contributions on the right-hand side and the three nonlinear third-order fluxes on the left-hand side. We close them by adopting the simplest dimensionally consistent local closure for both fluxes and dissipative terms:

$$
\begin{aligned}
\overline{\theta^{2} u_{z}} & =a_{\theta} L|\dot{L}| \partial_{z z} \overline{\theta^{2}}, \\
\overline{\left(u^{2}+p\right) u_{z}} & =a_{u_{z}} L|\dot{L}| \partial_{z z} \overline{u_{z}^{2}}, \\
\overline{\theta\left(u_{z}^{2}+p\right)} & =a_{\theta u_{z}} L|\dot{L}| \partial_{z z} \overline{\theta u_{z}}, \\
\epsilon_{\theta} & =b_{\theta} \overline{\theta^{2}} / \tau(z, t), \\
\epsilon_{v} & =b_{u_{z}} \overline{u_{z}^{2}} / \tau(z, t), \\
\epsilon_{\theta, u_{z}} & =b_{\theta u_{z}} \overline{\theta u_{z}} / \tau(z, t),
\end{aligned}
$$

where the typical time defining the dissipation rates is fixed by $\tau(z, t)=\sqrt{\overline{\overline{u_{z}^{2}}}} / L(t)$. Some comments are in order. First, we notice that the closure is now local but on the second-order moments, i.e., nonlocal for the evolution of mean profiles. Furthermore, out of the six free parameters, four can be handled quite robustly; all the three coefficients in front of the closure for third-order quantities are set $\mathcal{O}(1)$ using a first-order guess from the numerics: $a_{\theta}=0.2, a_{u_{z}}=0.3$, and $a_{\theta u_{z}}=0.8$. Moreover, the free parameter defining the intensity of the kinetic dissipation, $\epsilon_{v}$, is irrelevant in 2D (absence of direct energy cascade). The only two delicate free parameters are those defining the intensities of temperature and heat-flux dissipative terms, $\left[b_{\theta}, b_{\theta u_{z}}\right]$. In order to get a good agreement with the numerics we need some tuning. It is important to notice that both dissipative terms will develop a nontrivial vertical profile; i.e., they are not given by a simple bulk homogeneous parametrization.

The closed equations have been solved numerically, starting from the initial mean profiles obtained from the direct numerical simulations. The resulting partial differential equations in $(z, t)$ have been been solved with an implicit method in time, by discretizing the gradient and Laplacian operators in a suitable pentadiagonal (matrix) form. The resulting pentadiagonal matrix has then been inverted step by step. The expressions for $L(t)$ and $\dot{L}(t)$ have been evaluated numerically on the instantaneous profile using Eq. (7).

In Fig. 4 we show that the second-order closure is able to reproduce quantitatively the evolution of $L(t)$ for all cases. Let us notice that the global evolution of $L(t)$ is weakly dependent on the free parameters. Here, we show, for example, that keeping fixed $b_{\theta u_{z}}=5$ and at changing $b_{\theta} \in[0.35: 1.35]$ or keeping fixed $b_{\theta}=0.6$ and at changing $b_{\theta u_{z}} \in[4,5: 6.5]$ (top and bottom panels) one is always able to get a good agreement between the closure and the numerics. In the inset of the same figure we show for each choice of $\gamma$ the best fit value for both parameters, which gives also a good agreement with the whole temperature and heat-flux profiles at all times (see below).

In Figs. 6 and 7 we show the capability of the model to reproduce the heat flux vs temperature derivative locally for each $z$ along the whole profile, providing a sort of a posteriori effective eddy diffusivity. As one can see, the second-order

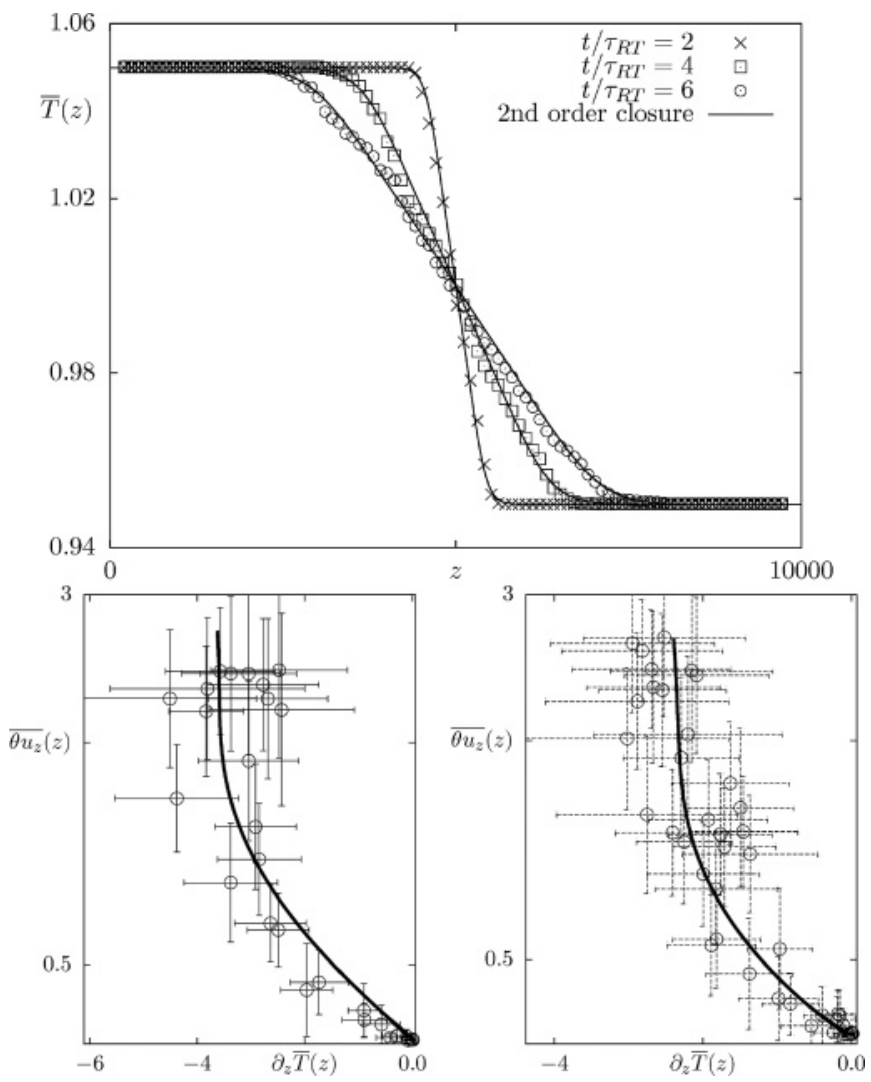

FIG. 6. Bottom panels: Check of the closure for the case with weak stratification (Run A). Circles: Numerical data. Solid lines: Second-order closure. Heat flux, $10^{4} \overline{\theta u_{z}}$, vs the temperature mean gradient, $10^{5} \partial_{z} \bar{T}$, for each location along the profile for two different times $t / t_{\mathrm{RT}}=3.8$ (left) and $t / t_{\mathrm{RT}}=5$ (right). Error bars are evaluated averaging over the configurations specified in Table I. Results from the second-order closure are given by the solid line with $\left[b_{\theta}, b_{\theta u_{z}}\right]=[1.45,4.5]$ and $b_{u_{z}}=0.03$. Top panel: Superposition of temperature profiles at three different times, $t / t_{\mathrm{RT}}=2,4,5.5$, with the corresponding curves from the second-order closure. 

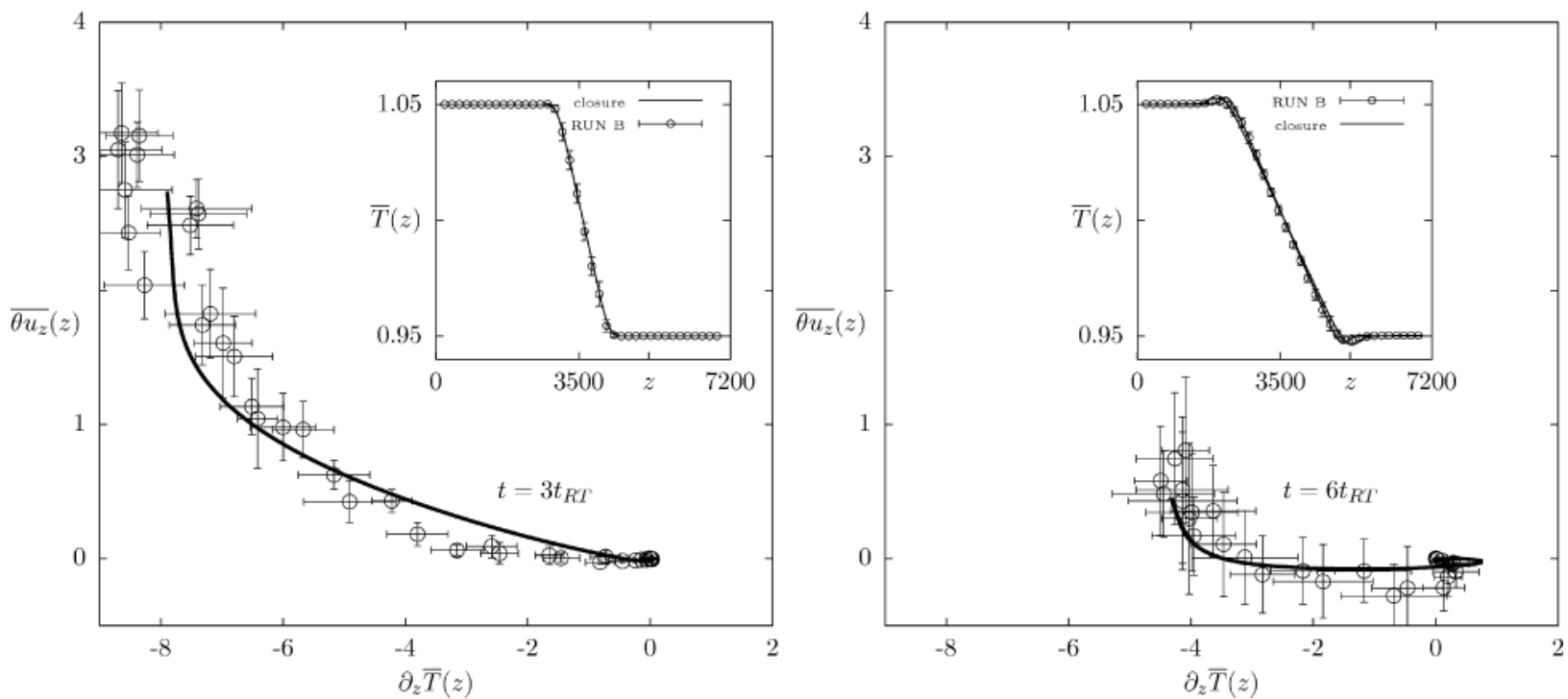

FIG. 7. Check of the second-order closure for the case with strong stratification (Run B). Circles: Numerical data. Solid line: Second-order closure. Heat flux, $10^{4} \overline{\theta u_{z}}$, vs the temperature mean gradient, $10^{5} \partial_{z} \bar{T}$, for each location along the profile, for two different times, $t=6 t_{\mathrm{RT}}$ (bottom panel) and $t=3 t_{\mathrm{RT}}$ (top panel). Solid lines: Second-order closure predictions $\left(\left[b_{\theta}, b_{\theta u_{z}}\right]=[0.35\right.$; 6.5]). Inset both panels: Matching between the temperature profile and the second-order closure.

closure is able to capture both the weak stratification case (Fig. 6) and the strong stratification case (Fig. 7). For the weak stratification case the effective eddy diffusivity is very close to the one predicted by using the Prandtl mixing length theory as proposed in Ref. [10] or to the one proposed by Spiegel [9] (not shown). For the strong stratification case, our model is able to capture also the long time behavior, even after the evolution has come to a halt due to the adiabatic gradient, where the heat flux has completely inverted sign (see bottom panel of Fig. 7). In the inset of the same figure we also show the capability of the second-order closure to reproduce the overshooting profile. As one can see the agreement is good. We also remark that from Eqs. (5) one may derive a dimensional closure neglecting all terms except the one at large scales, and assuming that temperature fluctuations are dominated by the global temperature jump: $\overline{\theta^{2}} \sim \Delta T$. From the third equation we derive $\overline{\theta u_{z}} \sim L{\overline{u_{z}^{2}}}^{1 / 2} \partial_{z} \bar{T}$ and from the second we derive $\overline{u_{z}^{2}} \sim g L^{2} \partial_{z} \bar{T}$, which-combined together-leads to Spiegel's closure: $\overline{\theta u_{z}} \sim L^{2}\left(\partial_{z} \bar{T}\right)^{1 / 2} \partial_{z} \bar{T}$.

In conclusion, we have performed state-of-the-art 2D numerical simulations using a LBM for turbulent convection driven by a RT instability in weakly and strongly stratified atmospheres. For the strongly stratified case, we are able to resolve the overshooting region with up to 800 grid points, something impossible to achieve with $3 \mathrm{D}$ direct numerical simulations because of the lack of computing power. We have presented a second-order closure to describe the evolution of mean fields able to capture both bulk properties and the overshooting observed at the boundary between the stable and the unstable regions. The closure is local in terms of fluctuations while keeping the evolution of the mean temperature profile exact. In order to apply the same closure to $3 \mathrm{D}$ cases one needs to take into account some possible nontrivial effects induced by the kinetic energy dissipation modeling, due to the presence of a forward energy cascade. As a result, also the parameter $b_{u_{z}}$ will become a relevant input in the model.

\section{ACKNOWLEDGMENTS}

We acknowledge useful discussions with G. Boffetta, A. Lawrie, and A. Wirth. We acknowledge access to QPACE and eQPACE during the bring-up phase of these systems. Parts of the simulations were also performed on CASPUR under HPC Grants (2009) and (2010).
[1] A. Siebesma, P. M. M. Soares, and J. Teixeira, J. Atmos. Sci. 64, 1230 (2007); A. Siebesma and J. W. M. Cuijpers, J. Atmos. Sci. 52, 650 (1995).

[2] V. M. Canuto and J. Christensen-Dalsgaard, Annu. Rev. Fluid Mech. 30, 167 (1998).

[3] H. G. Ludwig, F. Allard, and P. H. Hauschildt, Astron. Astrophys. 459, 599 (2006); R. Trampedach, Astrophys. Space Sci. 328, 213 (2010).
[4] W. G. Large, J. C. McWilliams, and S. C. Doney, Rev. Geophys. 32, 363 (1994).

[5] H. Burchard and K. Bolding, J. Phys. Oceanogr. 31, 1943 (2001).

[6] V. M. Canuto et al., J. Phys. Oceanogr. 32, 240 (2002).

[7] A. Wirth and B. Barnier, J. Phys. Oceanogr. 38, 803 (2008).

[8] E. Vitense, Zeitschrift fuer Astrophysik 32, 135 (1953). 
[9] E. A. Spiegel, Astrophys. J. 141, 1068 (1965); E. A. Spiegel and G. Veronis, ibid. 131, 442 (1960); E. A. Spiegel, ibid. 206, 536 (1976).

[10] G. Boffetta, F. De Lillo, and S. Musacchio, Phys. Rev. Lett. 104, 034505 (2010).

[11] N. J. Mueschke and O. Schilling, Phys. Fluids 21, 014106 (2009).

[12] P. Odier, J. Chen, M. K. Rivera, and R. E. Ecke, Phys. Rev. Lett. 102, 134504 (2009).

[13] S. Chandrasekhar, Hydrodynamic and Hydromagnetic Stability (Clarendon, Oxford, 1961).

[14] M.-A. Lafay, B. Le Creurer, and S. Gauthier, Europhys. Lett. 79, 64002 (2007).

[15] S. Gauthier (private communication).

[16] M. Chertkov, Phys. Rev. Lett. 91, 115001 (2003).

[17] A. Celani, A. Mazzino, and L. Vozella, Phys. Rev. Lett. 96, 134504 (2006).

[18] A. Scagliarini et al., Phys. Fluids 22, 055101 (2010).

[19] L. Biferale et al., Phys. Fluids 22, 115112 (2010).
[20] T. T. Clark et al., Phys. Fluids 15, 2413 (2003).

[21] J. R. Ristorcelli and T. T. Clark, J. Fluid Mech. 507, 213 (2004).

[22] W. H. Cabot and A. W. Cook, Nat. Phys. 2, 562 (2006).

[23] S. B. Dalziel et al., Phys. Fluids 20, 065106 (2008).

[24] S. I. Abarzhi, A. Gorobets, and K. R. Sreenivasan, Phys. Fluids 17, 081705 (2005).

[25] M. Sbragaglia et al., J. Fluid Mech. 628, 299 (2009).

[26] G. Goldrian et al. Comput. Sci. Eng. 10, 46 (2008).

[27] L. Biferale et al., Procedia Comput. Sci. 1, 1075 (2010).

[28] D. H. Sharp, Physica D 12, 3 (1984).

[29] D. Livescu et al., J. Turbul. 10, 1 (2009).

[30] G. Boffetta, A. Mazzino, S. Musacchio, and L. Vozella, Phys. Rev. E 79, 065301 (2009).

[31] L. D. Landau and E. M. Lifshitz, Fluid Mechanics (ButterworthHeinemann, Oxford, 1987).

[32] S. B. Pope, Turbulent Flows (Cambridge University Press, Cambridge, UK, 2000). 